\title{
On the proof of the thin sandwich conjecture in arbitrary dimensions
}

Cite as: J. Math. Phys. 58, 102502 (2017); https://doi.org/10.1063/1.4989573

Submitted: 09 June 2017 . Accepted: 11 October 2017 . Published Online: 27 October 2017

R. Avalos, F. Dahia, C. Romero, and J. H. Lira
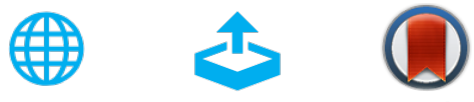

View Online

Export Citation

CrossMark

\section{ARTICLES YOU MAY BE INTERESTED IN}

Upper bound for diameter of cosmological black holes and nonexistence of black strings

Journal of Mathematical Physics 58, 102501 (2017); https://

doi.org/10.1063/1.5008259

\section{Does Levinson's theorem count complex eigenvalues?}

Journal of Mathematical Physics 58, 102101 (2017); https://

doi.org/10.1063/1.5004574

A new algorithm for computing branching rules and Clebsch-Gordan coefficients of unitary representations of compact groups

Journal of Mathematical Physics 58, 101702 (2017); https://

doi.org/10.1063/1.5004259

Where in the world is AIP Publishing?

Find out where we are exhibiting next 


\title{
On the proof of the thin sandwich conjecture in arbitrary dimensions
}

\author{
R. Avalos, ${ }^{\text {a) }}$ F. Dahia, ${ }^{\text {a) }}$ C. Romero, ${ }^{\text {a) }}$ and J. H. Lira ${ }^{\text {a) }}$ \\ Departamento de Física, Universidade Federal da Paraíba, Caixa Postal 5008, \\ 58059-970 João Pessoa, PB, Brazil
}

(Received 9 June 2017; accepted 11 October 2017; published online 27 October 2017)

\begin{abstract}
In this paper we show the validity, under certain geometric conditions, of Wheeler's thin sandwich conjecture for higher dimensional theories of gravity. We extend the results for the 3-dimensional case in Phys. Rev. D 48, 3596-3599 (1993) in two ways. On the one hand, we show that the results presented in Phys. Rev. D 48, 3596-3599 (1993) are valid in arbitrary dimensions, and on the other hand, we show that the geometric hypotheses needed for the proofs can always be satisfied, which constitutes in itself a new result for the 3 -dimensional case. In this way, we show that on any compact $n$-dimensional manifold, $n \geq 3$, there is an open set in the space of all possible initial data where the thin sandwich problem is well-posed. Published by AIP Publishing. https://doi.org/10.1063/1.4989573
\end{abstract}

\section{INTRODUCTION}

As is well known, the Cauchy problem for general relativity (GR) consists in finding a solution of the Einstein equations in a 4-dimensional Lorentzian manifold, which satisfies some prescribed initial conditions on a 3-dimensional Riemannian hypersurface. This can be understood as studying whether we can propagate some initial space-like hypersurface, such that the resulting space-time satisfies the Einstein equations. This problem has long been studied and there are results which show that general relativity has a well-posed Cauchy problem for initial data satisfying some constraint equations. ${ }^{2} \mathrm{~A}$ detailed review on this topic can be found in Refs. 3 and 4. These constraint equations imply that we cannot arbitrarily give the initial data set for the Cauchy problem, motivating the study of these equations so as to determine under what conditions it has a solution and what part of these data can in fact be given arbitrarily on the initial manifold. It is customary to regard this system as a system of partial differential equations (PDE) for a Riemannian metric and for some $(0,2)$ symmetric tensor field defined on this Riemannian hypersurface, which in the end will play the role of the extrinsic curvature. We now know that under some hypotheses on the topology of the space-like manifold, we can specify a conformal metric to the physical one (that is, the one which will solve the constraints and hence will have a development in space-time) and the trace of the second fundamental form and then get a well-posed system for the remaining undetermined quantities. ${ }^{3}$ Another way to look at this problem was proposed by Wheeler. His idea was to consider space-time as a curve in what he called Superspace. Intuitively, given a 3-dimensional manifold $M$, the Superspace $S(M)$ related to it would be the space of geometries that can be defined on $M$. In this way, a point in $S(M)$ is regarded as an equivalence class $(M,[g])$, represented by a pair $(M, g)$ with $g$ a Riemannian metric defined on $M$, where two Riemannian metrics are considered equivalent if they are related to each other by a diffeomorphism via pullback. A detailed review on this structure can be found in Ref. 5 . With this in mind, we can think of space-time as a curve in Superspace. In this context, Wheeler proposed the Thin Sandwich Problem (TSP), ${ }^{6}$ where the idea is to give as initial data a Riemannian metric $g$ and a tangent vector $\partial_{t} g$ to $(M, g)$ and then study whether we can solve the constraint

\footnotetext{
a)Electronic addresses: rodrigo.avalos@fisica.ufpb.br; fdahia@fisica.ufpb.br; cromero@ fisica.ufpb.br; and jorge.lira@mat. ufc.br
} 
equations for these initial data. If we can, then these initial data have a unique Cauchy development in space-time. This means that there would be a unique curve in Superspace satisfying these initial conditions and compatible with the Einstein equations. This problem has been recently investigated by some authors. ${ }^{1,7,8}$ In this paper, we will be particularly interested in the results obtained by Bartnik and Fodor, who, for the 3-dimensional case, which is the arena of classical general relativity, have established sufficient conditions for the TSP to be well-posed. ${ }^{1}$ More precisely, they have shown that given some free data $\left(g_{0}, \dot{g}_{0}, \epsilon_{0}, S_{0}\right)$ satisfying some specific geometric conditions and for which a solution of the constraints in their thin-sandwich formulation exists, there is a neighbourhood of this free data set where the TSP is well-posed. Even though this result mainly relies on both elliptic theory and an implicit function argument, which do not generally depend on the dimension, in the proofs they explicitly take advantage of the fact that they are working in 3-dimensions to manipulate expressions in a way which is not practical in arbitrary dimensions. But since, just as for the evolution problem in GR, the setting of the constraint equations in its classical formulation does not strongly depend on the dimension, it would be expected that the results presented in Ref. 1 should extend to arbitrary dimensions $(n \geq 3)$. We will show that this is actually true and that there is in fact an $n$-dimensional analog of the Bartnik-Fodor theorem. Also, in Ref. 1, in order to show that there are reference solutions for the constraint equations where their main theorem applies, they produce an example using the initial data induced by the spatially compactified Friedmann-Robertson-Walker cosmological solution with $k=-1$, where all the conditions needed for this theorem are satisfied. Nevertheless, it is not shown that on any compact 3-dimensional manifold a reference solution exists. In this paper, we show that this last statement actually holds, that is, on any compact $n$-dimensional manifold, $n \geq 3$, there are reference solutions of the constraint equations satisfying all the hypotheses needed to apply the implicit function argument. In this way, we will be concerned with the local well-posedness of the TSP, where by this we mean that we will show that in a neighbourhood of free data with specific properties, the TSP has a unique solution. It should be stressed that we do not expect this problem to be well-posed for arbitrary data. For instance, following an argument presented by Belasco and Ohanian in Ref. 7, if we choose data $(g, \dot{g}, \epsilon, S)$ such that $2 \epsilon-R(g)>0$ and $\dot{g}=£_{X} g$ for any smooth vector field $X$, then no solution for the TSP can exist on a compact (without boundary) and connected manifold $M$.

We would also like to draw the reader's attention to Ref. 8, where a generalization of Ref. 1 is presented which includes models for the matter fields in a more realistic way. Even though we did not follow this approach, it is worth emphasizing that the framework and techniques presented in this paper could provide interesting future developments on higher-dimensional TSPs as well.

\section{STATEMENT OF THE PROBLEM}

The Cauchy problem for general relativity (GR) consists in the following. Given an initial data set $(M, g, K)$ where $M$ is an $n$-dimensional smooth Riemannian manifold with metric $g$ and $K$ is a symmetric second rank tensor field, a development of this initial data set is a space-time $(V, \bar{g})$, such that there exists an embedding into $\iota: M \mapsto V$ with the following properties:

(i) The metric $g$ is the pullback of $\bar{g}$ by the embedding $\iota$, that is, $\iota^{*} \bar{g}=g$.

(ii) The image by $\iota$ of $K$ is the second fundamental form of $\iota(M)$ as a submanifold of $(V, g)$.

In the Cauchy problem for GR, we look for a development of an initial data set such that the resulting space-time satisfies the Einstein equations. It is customarily assumed that $V=M \times \mathbb{R}$. Since this is a consequence of global hyperbolicity, we do not regard it as a physical obstruction, and thus we will adopt this usual setting.

At this point, to study the Cauchy problem, it is customary to consider an $(n+1)$-dimensional space-time $(V, \bar{g})$ and then make an " $(n+1)$-splitting" for the metric $\bar{g}$. This means that we consider local co-frames where we can write the metric $\bar{g}$ in a convenient way, such that we have a "spacetime splitting." In order to do this, a vector field $\beta$, which is constructed so as to be tangent to each hypersurface $M \times\{t\}$, is used to define the following local frame: 


$$
\begin{aligned}
e_{i} & =\partial_{i}, \quad i=1, \ldots, n, \\
e_{0} & =\partial_{t}-\beta
\end{aligned}
$$

and its dual coframe

$$
\begin{aligned}
\theta^{i} & =d x^{i}+\beta^{i} d t, i=1, \ldots, n, \\
\theta^{0} & =d t .
\end{aligned}
$$

Then we can write the metric $\bar{g}$ in the following way:

$$
\bar{g}=-N^{2} \theta^{0} \otimes \theta^{0}+g_{i j} \theta^{i} \otimes \theta^{j},
$$

where the function $N$ is a positive function referred to as the lapse function, while the vector field $\beta$ is called the shift vector.

In this adapted frame, the second fundamental form on each $M \times\{t\}$ takes the form

$$
K_{i j}=\frac{1}{2 N}\left(\partial_{t} g_{i j}-\left(\nabla_{i} \beta_{j}+\nabla_{j} \beta_{i}\right)\right),
$$

where $\nabla$ denotes the induced connection in $M$ compatible with the induced metric $g$.

As we have already noted, the possibility of finding an Einsteinian development of an initial data set depends on whether the following set of constraint equations are satisfied by these initial data:

$$
\begin{aligned}
R_{g}-|K|_{g}^{2}+\left(\operatorname{tr}_{g} K\right)^{2} & =2 \epsilon, \\
\operatorname{div} K-\nabla \operatorname{tr}_{g} K & =S,
\end{aligned}
$$

where $(\epsilon, S)$ denote the induced energy and momentum densities on $M$, respectively, $R_{g}$ represents the scalar curvature of $g,|\cdot|_{g}$ denotes the pointwise-tensor norm in the metric $g$, and $\operatorname{div} K \operatorname{denotes}$ the divergence of $K$. These constraint equations are posed on an $n$-dimensional manifold $M$ and are imposed by the $(n+1)$-dimensional Einstein equations (see, for instance, Ref. 3$)$. In coordinates, these equations become

$$
\begin{aligned}
R_{g}-K^{i j} K_{i j}+\left(K_{i}^{i}\right)^{2} & =2 \epsilon, \\
\nabla_{j} K_{i}^{j}-\nabla_{i} K_{j}^{j} & =S_{i} .
\end{aligned}
$$

These equations are considered on a particular initial hypersurface $M \cong M \times\{t\}$, for example, in the hypersurface defined by $t=0$. If our initial data set $(M, g, K)$ satisfies these constraints, then, for many sources of interest, it can be shown that there is an Einsteinian development in our space-time $V .^{3}$

Equations (4) and (5) are generally posed as a set of equations for $g$ and $K$. In the context of Wheeler's TSP, these equations are looked as equations for $N$ and $\beta$. In order to do this, we use (1) to express (4) and (5) in terms of the lapse and shift and then look for solutions with some prescribed data $(g, \dot{g}, \epsilon, S)$, where $\dot{g}=\partial_{t} g$.

In the scenario of the TSP, suppose that, given some prescribed data $(g, \dot{g}, \epsilon, S)$, we have a solution $(N, \beta)$ for the constraint equations. Furthermore, suppose this solution satisfies $2 \epsilon-R_{g} \neq 0$ over all $M$. Then, introducing (1) in (4) we can equate the lapse function in terms of the shift vector and the prescribed data. After doing this, we obtain

$$
N=\sqrt{\frac{\left(\operatorname{tr}_{g} \gamma\right)^{2}-|\gamma|_{g}^{2}}{2 \epsilon-R_{g}}},
$$

where the tensor $\gamma$ has components

$$
\gamma_{i j}=\frac{1}{2}\left(\dot{g}_{i j}-\left(\nabla_{i} \beta_{j}+\nabla_{j} \beta_{i}\right)\right) .
$$

It should be noted that in (6) we have chosen the positive sign for the square root, since this choice, which corresponds to the choice of positive lapse, is related to the choice of a space-time foliation which evolves to the future, whereas the negative sign would represent a foliation evolving to the past. Furthermore, a few comments on the individual signs of the numerator and denominator are in order. 
First of all, note that if $M$ is connected, then the condition $2 \epsilon-R_{g} \neq 0$ at each point of $M$ implies that $2 \epsilon-R_{g}$ has a definite sign on $M$. It should be noted that later on we will impose the condition $2 \epsilon-R_{g}>0$ for a reference solution of the constraint equations, in a neighbourhood of which we will study the TSP. This condition, which imposes an energy constraint, forces the numerator in (6) to be strictly positive and, furthermore, requires that $\operatorname{tr}_{\mathrm{g}} \gamma \neq 0 \forall p \in M$. Using (1) and the definition of $\gamma$, we see that if $M$ is connected, this implies that $\tau \doteq \operatorname{tr}_{\mathrm{g}} \mathrm{K}$ has a definite sign all over $M$. That is, if this initial data set has an embedding into a space-time satisfying the Einstein equations, then the hypersurface $M \times\{0\} \cong M$ will be an embedded hypersurface whose mean curvature has a definite sign. This fact carries a clear physical interpretation: the sign of the mean curvature is related to whether the future pointing unit normals are diverging from the hypersurface or converging, representing, respectively, an expanding or contracting space evolving in space-time.

Now, replacing (6) in (5) shows that the shift vector satisfies the following equation:

$$
\nabla_{i}\left(\sqrt{\frac{2 \epsilon-R_{g}}{\left(\operatorname{tr}_{g} \gamma\right)^{2}-|\gamma|_{g}^{2}}}\left(\gamma_{j}^{i}-\delta_{j}^{i} \operatorname{tr}_{g} \gamma\right)\right)=S_{j},
$$

that is,

$$
\operatorname{div}\left(\sqrt{\frac{2 \epsilon-R_{g}}{\left(\operatorname{tr}_{g} \gamma\right)^{2}-|\gamma|_{g}^{2}}}\left(\gamma-\operatorname{tr}_{g} \gamma g\right)\right)=S .
$$

We have a converse procedure to the one just described. That is, if, for a given initial data set $(g, \dot{g}, \epsilon, S),(8)$ is well-posed and $\beta$ is a solution of (8), then taking (6) as a definition, the lapse will satisfy (4).

It is worth pointing out that Eq. (9) has a variational origin (see Refs. 1 and 7). In particular, the first detailed treatment of the thin-sandwich problem was made using this variational formulation. ${ }^{7}$ There, some uniqueness and non-existence results were shown, including a global uniqueness result (see also Ref. 8).

We can now state the problem we want to study here. Given a solution $(N, \beta)$ of the constraint equations obtained from some given data $(g, \dot{g}, \epsilon, S)$, can we obtain a solution of the constraint equations for data "sufficiently near" of these given data? We will first show that, under certain hypotheses, this can be answered affirmatively and then that these hypotheses can always be satisfied by some reference solution on any compact $n$-dimensional manifold $\forall n \geq 3$. Note that proving that for any set of initial data sufficiently near to $(g, \dot{g}, \epsilon, S)$ there is a unique solution of the constraint equations, also proves that if the associated evolution problem is well-posed, then for these data there exists a unique Cauchy development in space-time, and this, in turn, would prove a restricted form of Wheeler's thin sandwich conjecture.

Before going further, it would be appropriate to remark that when we say that the quantity $\dot{g}$ is a given datum, we mean that some symmetric $(0,2)$ tensor field on $M$ is given and that with this tensor field we construct $K$ from (1), taking this tensor field as $\partial_{t} g_{i j}$. Then if we have a solution for the Cauchy problem, this tensor field will coincide with $\partial_{t} g_{i j}$ on $M \times\{0\}$

\section{MAIN RESULTS}

As we have stated above, we need to study whether any initial data set $(g, \dot{g}, \epsilon, S)$ sufficiently near to a reference solution of the constraint equations also satisfies the constraint equations. In order to do this, we can concentrate ourselves to answer this question just for the set of Eqs. (8). In order to proceed, we will assume $M$ to be compact (without boundary) and write this set of non-linear PDE for the shift vector in the following way. Let

$$
H_{s}\left(T_{q}^{p}(M)\right), \quad s>\frac{n}{2}, s>2,
$$

be the space of $(p, q)$-tensor fields in $M$ with local components in the Sobolev space $H_{s}(\Omega)$, where $\Omega$ is an open subset of $\mathbb{R}^{n}$. Denote

$$
\mathcal{E}_{1} \doteq H_{s+3}\left(T_{2}^{0} M\right) \times H_{s+1}\left(T_{2}^{0} M\right) \times H_{s+1}(M) \times H_{s}\left(T_{1}^{0} M\right)
$$


which is a Banach space with the norm $\|\cdot\|_{\mathcal{E}_{1}}: \mathcal{E}_{1} \rightarrow \mathbb{R}$ given by

$$
\|(g, \dot{g}, \epsilon, S)\|_{\mathcal{E}_{1}}=\|g\|_{H_{s+3}}+\|\dot{g}\|_{H_{s+1}}+\|\epsilon\|_{H_{s+1}}+\|S\|_{H_{s}}
$$

and let

$$
\mathcal{E}_{2} \doteq H_{s+2}\left(T_{0}^{1} M\right) \text { and } \mathcal{F} \doteq H_{s}\left(T_{1}^{0} M\right)
$$

Now suppose that for given data $\psi_{0} \doteq\left(g_{0}, \dot{g}_{0}, \epsilon_{0}, S_{0}\right) \in \mathcal{E}_{1}$ we have a solution $\beta_{0} \in \mathcal{E}_{2}$. Then, the continuity of all the maps involved guarantees that (8) is well-defined in a neighborhood $\mathcal{U}$ of $\left(\psi_{0}\right.$, $\left.\beta_{0}\right)$ in $\mathcal{E}_{1} \times \mathcal{E}_{2}$. With this in mind, we define the map

$$
\Phi: \mathcal{U} \subset \mathcal{E}_{1} \times \mathcal{E}_{2} \rightarrow \mathcal{F}
$$

given by

$$
\Phi(\psi, \beta) \doteq \operatorname{div}\left(\sqrt{\frac{2 \epsilon-R_{g}}{\left(\operatorname{tr}_{g} \gamma\right)^{2}-|\gamma|_{g}^{2}}}\left(\gamma-\operatorname{tr}_{g} \gamma g\right)\right)-S,
$$

where we have denoted $\psi=(g, \dot{g}, \epsilon, S)$, and we are using $\beta$ to denote the shift. Then (8) can be written as

$$
\Phi(\psi, \beta)=0 .
$$

Now our problem reduces to the following: we want to see if there are open sets $\mathcal{V} \subset \mathcal{E}_{1}, \mathcal{W} \subset \mathcal{E}_{2}$, with $\psi_{0} \in \mathcal{V}$ and $\beta_{0} \in \mathcal{W}$, and a unique map

$$
g: \mathcal{V} \rightarrow \mathcal{W}
$$

such that

$$
\Phi(\psi, g(\psi))=0 \text { for all } \psi \in \mathcal{V} .
$$

Notice that, in this case, $\beta=g(\psi) \in \mathcal{W}$ would be the solution to our problem. In order to address this issue, we intend to use the implicit function theorem. Hence, we need to show that

$$
L=\left.\frac{\delta \Phi}{\delta \beta}\right|_{\left(\psi_{0}, \beta_{0}\right)}: \mathcal{E}_{2} \rightarrow \mathcal{F}
$$

is an isomorphism. This is precisely the procedure followed in Ref. 1 in the 3-dimensional case. We will extend their results for arbitrary dimensions $(n \geq 3)$. Using (10), we compute

$$
L \mathcal{Y}=\left.\frac{\delta \Phi}{\delta \beta}\right|_{(\psi, \beta)}=\operatorname{div}\left(\frac{1}{N}\left(\operatorname{div} \mathcal{Y} g-{ }^{S} \nabla \mathcal{Y}-\frac{1}{2 \epsilon-R_{g}}\langle\pi, \nabla \mathcal{Y}\rangle \pi\right)\right),
$$

where $\pi$ is the tensor,

$$
\pi \doteq \frac{1}{N}\left(\gamma-\operatorname{tr}_{g} \gamma g\right)=K-\operatorname{tr}_{g} K g,
$$

which represents the conjugate momentum to $g$ in the Hamiltonian picture of GR, and

$$
{ }^{S} \nabla_{i} \mathcal{Y}_{j}=\frac{1}{2}\left(\nabla_{i} \mathcal{Y}_{j}+\nabla_{j} \mathcal{Y}_{i}\right)
$$

We will study the properties of the linearized operator $L$. First of all, it is clear that $L$ is a linear second order operator. We now have the following proposition.

Proposition 1. If $\pi$ is a definite operator all over $M$, then the linear operator $L$ is elliptic.

Proof. The first thing we need to do is to compute the symbol of the linear operator $L$. We easily verify that the symbol of $L$ is given by

$$
(\sigma(L)(\xi) \cdot \mathcal{Y})^{j}=\frac{1}{N}\left(\frac{1}{2} \xi^{j} \xi_{k}-\frac{1}{2}|\xi|_{g}^{2} \delta_{k}^{j}-\frac{1}{2 \epsilon-R_{g}} \pi^{i j} \xi_{i} \pi_{k}^{\ell} \xi_{\ell}\right) \mathcal{Y}^{k},
$$

for all $\xi \in \Gamma\left(T^{*} M\right)$ and $\mathcal{Y} \in \Gamma(T M)$. Hence

$$
\begin{aligned}
\langle\sigma(L)(\xi) \cdot \mathcal{Y}, \xi\rangle & =(\sigma(L)(\xi) \cdot \mathcal{Y})^{j} \xi_{j}=\frac{1}{N}\left(\frac{1}{2}|\xi|_{g}^{2}\langle\xi, \mathcal{Y}\rangle-\frac{1}{2}|\xi|_{g}^{2}\langle\xi, \mathcal{Y}\rangle-\frac{1}{2 \epsilon-R_{g}} \pi(\xi, \xi) \pi(\xi, \mathcal{Y})\right) \\
& =-\frac{1}{2 \epsilon-R_{g}} \pi(\xi, \xi) \pi(\xi, \mathcal{Y}) .
\end{aligned}
$$


Suppose $\mathcal{Y} \in T_{p} M$ such that $\sigma(L)(\xi) \cdot \mathcal{Y}=0$ for some $\xi \neq 0$. Then

$$
\pi(\xi, \xi) \pi(\xi, \mathcal{Y})=0
$$

for some $\xi \neq 0$. Since by assumption $\pi$ is definite and hence non-degenerate, this implies that $\pi(\xi, \mathcal{Y})$ $=0, \xi \neq 0$. Using this information in (15), we get that if $\mathcal{Y}$ is in the null space of $\sigma(L)(\xi)$, then $\mathcal{Y}$ $=\left\langle\frac{\xi}{|\xi|_{g}^{2}}, \mathcal{Y}\right\rangle \xi$. This two conditions, together with the fact that $\pi$ is non-degenerate, imply that $\mathcal{Y}=0$. Thus $L$ is elliptic.

It is interesting to note that the condition on $\pi$ being a definite operator has one particular consequence with a clear physical interpretation. Note that $\pi$ being definite imposes a condition on $\operatorname{tr}_{\mathrm{g}} K$, since, using (14), we get that $\operatorname{tr}_{\mathrm{g}} \mathrm{K}=\frac{1}{1-n} \operatorname{tr}_{\mathrm{g}} \pi$. Also, note that $\pi$ defines an operator $\pi^{\sharp}$ on vector fields, given in components by $\pi^{\sharp}(X)^{i} \doteq \pi_{j}^{i} X^{j}$. Note that the symmetry of $\pi$ shows that $\pi^{\sharp}$ defines a self-adjoint operator (with respect to $g$ ) on each tangent space. That is, $\left\langle v, \pi^{\sharp}(w)\right\rangle=\left\langle\pi^{\sharp}(v), w\right\rangle$ for all $v, w \in T_{p} M$ and $p \in M$. This means that, at each point, there is a $g$-orthonormal basis diagonalizing $\pi$. Using such basis in order to compute $\operatorname{tr}_{\mathrm{g}} \pi$, we see that the trace is the sum of the eigenvalues of $\pi$, and thus, that if $\pi$ is definite, the trace must be either strictly positive or strictly negative. This implies that if $\pi$ is definite on $M$, then, if $M$ is connected, $\operatorname{tr}_{\mathrm{g}} K$ must have a constant $\operatorname{sign}$ on $M$ and cannot be zero. Now, if a given initial data set $(g, K)$ satisfying this condition on the trace of $K$ has a development into a space-time $V$, then the embedded hypersurface $M \times\{0\} \cong M$ has mean curvature with a definite sign all over the hypersurface. This, again, can be interpreted as telling us that the whole hypersurface is either expanding or contracting in its space-time evolution (at least for short times).

From now on, we will suppose that $\pi$ gives a definite operator at every point of $M$ so that the last proposition holds. Having in mind that our aim is to establish sufficient conditions so that $L$ is an isomorphism, the ellipticity condition just established shows that what we need to do is to show the injectivity of both $L$ and its formal adjoint $L^{*}$. A straightforward computation, using integration by parts, gives us that $L$ is (formally) self-adjoint. This means that for all smooth vector fields $\mathcal{Y}, \mathcal{Z}$, the following holds:

$$
\int_{M}\langle L \mathcal{Y}, \mathcal{Z}\rangle \mathrm{d} M_{g_{0}}=\int_{M}\langle\mathcal{Y}, L \mathcal{Z}\rangle \mathrm{d} M_{g_{0}}
$$

where $\mathrm{d} M_{g_{0}}$ is the Riemannian volume element in $M$ induced by the metric $g_{0}$. Thus, if $\pi$ is a definite operator on $M$, then $L$ is a (formally) self-adjoint elliptic operator, and what we need to establish is its injectivity, which is the content of the following proposition.

Proposition 2. Consider a reference solution $(\psi, \beta)$ for the TSP on a compact $n$-dimensional manifold $M$ satisfying that (i) $\pi$ is a definite operator on $M$, (ii) $2 \epsilon-R_{g}>0$ on $M$, (iii) given a function $\mu$, the equation

$$
{ }^{s} \nabla \mathcal{Y}=\mu K
$$

has only the solution $\mathcal{Y}=0, \mu=0$. Then $L$ is injective.

Proof. Recall that

$$
L \mathcal{Y}=\operatorname{div}\left(\frac{1}{N}\left(\operatorname{div} \mathcal{Y} g-\frac{1}{2} £_{\mathcal{Y}} g-\frac{1}{2 \varepsilon-R}\langle\pi, \nabla \mathcal{Y}\rangle \pi\right)\right) .
$$

Let $\Omega$ be a relatively compact open subset in $M$ and let $\eta \in C_{0}^{\infty}(\Omega)$ with $\eta \equiv 1$ in $\Omega^{\prime} \subset \Omega$. Denoting $\mathcal{Z}=\eta \mathcal{Y}$, one obtains

$$
\begin{aligned}
\langle L \mathcal{Y}, \mathcal{Z}\rangle & =Z^{j} \nabla_{i}\left(\frac{1}{N}\left(\operatorname{div} \mathcal{Y} \delta_{j}^{i}-\frac{1}{2}\left(\nabla_{j} \mathcal{Y}^{i}+\nabla^{i} \mathcal{Y}_{j}\right)-\frac{1}{2 \varepsilon-R}\langle\pi, \nabla \mathcal{Y}\rangle \pi_{j}^{i}\right)\right) \\
& =\nabla_{i}\left(\frac{1}{N}\left(\operatorname{div} \mathcal{Y} \delta_{j}^{i}-\frac{1}{2}\left(\nabla_{j} \mathcal{Y}^{i}+\nabla^{i} \mathcal{Y}_{j}\right)-\frac{1}{2 \varepsilon-R}\langle\pi, \nabla \mathcal{Y}\rangle \pi_{j}^{i}\right) Z^{j}\right) \\
& -\frac{1}{N}\left(\operatorname{div} \mathcal{Y} \delta_{j}^{i}-\frac{1}{2}\left(\nabla_{j} \mathcal{Y}^{i}+\nabla^{i} \mathcal{Y}_{j}\right)-\frac{1}{2 \varepsilon-R}\langle\pi, \nabla \mathcal{Y}\rangle \pi_{j}^{i}\right) \nabla_{i} Z^{j}
\end{aligned}
$$


which yields

$$
\begin{aligned}
\langle L \mathcal{Y}, \mathcal{Z}\rangle & =\operatorname{div}\left(\frac{1}{N}\left(\mathcal{Z} \operatorname{div} \mathcal{Y}-\frac{1}{2} f_{\mathcal{Y}} g(\mathcal{Z}, \cdot)-\frac{1}{2 \varepsilon-R}\langle\pi, \nabla \mathcal{Y}\rangle \pi(\mathcal{Z}, \cdot)\right)\right) \\
& -\frac{1}{N}\left(\operatorname{div} \mathcal{Y} \operatorname{div} \mathcal{Z}-\left\langle{ }^{S} \nabla \mathcal{Y}, \nabla \mathcal{Z}\right\rangle-\frac{1}{2 \varepsilon-R}\langle\pi, \nabla \mathcal{Y}\rangle\langle\pi, \nabla \mathcal{Z}\rangle\right) .
\end{aligned}
$$

We conclude that

$$
\begin{aligned}
\int_{\Omega}\langle L \mathcal{Y}, \mathcal{Z}\rangle \mathrm{d} M & =\int_{\partial \Omega} \frac{1}{N}\left(\langle\mathcal{Z}, v\rangle \operatorname{div} \mathcal{Y}-\frac{1}{2} £_{\mathcal{Y}} g(\mathcal{Z}, v)-\frac{1}{2 \varepsilon-R}\langle\pi, \nabla \mathcal{Y}\rangle\langle\pi(\mathcal{Z}), v\rangle\right) \mathrm{d} \partial M \\
& -\int_{\Omega} \frac{1}{N}\left(\operatorname{div} \mathcal{Y} \operatorname{div} \mathcal{Z}-\left\langle{ }^{S} \nabla \mathcal{Y}, \nabla \mathcal{Z}\right\rangle-\frac{1}{2 \varepsilon-R}\langle\pi, \nabla \mathcal{Y}\rangle\langle\pi, \nabla \mathcal{Z}\rangle\right) \mathrm{d} M,
\end{aligned}
$$

where $v$ stands for the outward normal to $\partial M$. Since the integrand of the first term in the right-hand side vanishes at the boundary, it follows that if $L \mathcal{Y}=0$, then

$$
\int_{\Omega} \frac{1}{N}\left(\operatorname{div} \mathcal{Y} \operatorname{div} \mathcal{Z}-\left\langle{ }^{S} \nabla \mathcal{Y}, \nabla \mathcal{Z}\right\rangle-\frac{1}{2 \varepsilon-R}\langle\pi, \nabla \mathcal{Y}\rangle\langle\pi, \nabla \mathcal{Z}\rangle\right) \mathrm{d} M=0 .
$$

In particular on $\Omega^{\prime} \subset \Omega$, we have

$$
\int_{\Omega^{\prime}} \frac{1}{N}\left(\left\langle{ }^{S} \nabla \mathcal{Y}, \nabla \mathcal{Y}\right\rangle-(\operatorname{div} \mathcal{Y})^{2}+\frac{1}{2 \varepsilon-R}\langle\pi, \nabla \mathcal{Y}\rangle^{2}\right) \mathrm{d} M=0 .
$$

Now define

$$
I \doteq\left\langle{ }^{S} \nabla \mathcal{Y}, \nabla \mathcal{Y}\right\rangle-(\operatorname{div} \mathcal{Y})^{2}+\frac{1}{2 \varepsilon-R}\langle\pi, \nabla \mathcal{Y}\rangle^{2}
$$

Since $(\psi, \beta)$ gives a reference solution for the constraint equations, using (4) we know that

$$
\frac{(\operatorname{tr} K)^{2}-|K|_{g}^{2}}{2 \epsilon-R}=1
$$

We note that

$$
\left\langle{ }^{S} \nabla \mathcal{Y}, \nabla \mathcal{Y}\right\rangle=\left\langle{ }^{S} \nabla \mathcal{Y},{ }^{S} \nabla \mathcal{Y}\right\rangle=\left|{ }^{S} \nabla \mathcal{Y}\right|_{g}^{2}
$$

and

$$
\langle\pi, \nabla \mathcal{Y}\rangle=\left\langle\pi,{ }^{S} \nabla \mathcal{Y}\right\rangle
$$

We also have

$$
\operatorname{div} \mathcal{Y}=g^{i j} \nabla_{i} \mathcal{Y}_{j}=\langle g, \nabla \mathcal{Y}\rangle
$$

and

$$
\langle K, \nabla \mathcal{Y}\rangle=\left\langle K,{ }^{S} \nabla \mathcal{Y}\right\rangle .
$$

Note that since $\pi$ is definite, then $|K|_{g} \neq 0$. Thus we denote

$$
\lambda=\frac{1}{|K|_{g}^{2}}\langle\nabla \mathcal{Y}, K\rangle
$$

and rewrite (21) above as

$$
\begin{aligned}
I & =\left|{ }^{S} \nabla \mathcal{Y}-\lambda K\right|_{g}^{2}+2 \lambda\left\langle{ }^{S} \nabla \mathcal{Y}, K\right\rangle-\lambda^{2}|K|_{g}^{2}-(\operatorname{div} \mathcal{Y})^{2}+\frac{1}{2 \varepsilon-R}\langle\pi, \nabla \mathcal{Y}\rangle^{2} \\
& =\left|{ }^{S} \nabla \mathcal{Y}-\lambda K\right|_{g}^{2}+2 \frac{1}{|K|_{g}^{2}}\left\langle{ }^{S} \nabla \mathcal{Y}, K\right\rangle^{2}-\frac{1}{|K|_{g}^{2}}\left\langle{ }^{S} \nabla \mathcal{Y}, K\right\rangle^{2}-(\operatorname{div} \mathcal{Y})^{2}+\frac{1}{2 \varepsilon-R}\langle\pi, \nabla \mathcal{Y}\rangle^{2} .
\end{aligned}
$$

However, since

$$
\pi=K-\operatorname{tr} K g
$$

and

$$
\langle\pi, \nabla \mathcal{Y}\rangle=\langle K, \nabla \mathcal{Y}\rangle-\operatorname{tr} K\langle g, \nabla \mathcal{Y}\rangle=\left\langle{ }^{S} \nabla \mathcal{Y}, K\right\rangle-\operatorname{tr} K \operatorname{div} \mathcal{Y},
$$


we get that

$$
\begin{aligned}
I & =\left|{ }^{S} \nabla \mathcal{Y}-\lambda K\right|_{g}^{2}+\frac{1}{|K|_{g}^{2}}\left\langle{ }^{S} \nabla \mathcal{Y}, K\right\rangle^{2}-(\operatorname{div} \mathcal{Y})^{2} \\
& +\frac{1}{2 \varepsilon-R}\left(\left\langle{ }^{S} \nabla \mathcal{Y}, K\right\rangle^{2}-2 \operatorname{tr} K \operatorname{div} \mathcal{Y}\left\langle{ }^{S} \nabla \mathcal{Y}, K\right\rangle+(\operatorname{tr} K)^{2}(\operatorname{div} \mathcal{Y})^{2}\right) .
\end{aligned}
$$

Using again the fact that

$$
2 \varepsilon-R=(\operatorname{tr} K)^{2}-|K|_{g}^{2}
$$

we have

$$
\begin{aligned}
I & =\left|{ }^{S} \nabla \mathcal{Y}-\lambda K\right|_{g}^{2}+\left(\frac{1}{|K|_{g}^{2}}+\frac{1}{(\operatorname{tr} K)^{2}-|K|_{g}^{2}}\right)\left\langle{ }^{S} \nabla \mathcal{Y}, K\right\rangle^{2}+\left(\frac{(\operatorname{tr} K)^{2}}{(\operatorname{tr} K)^{2}-|K|_{g}^{2}}-1\right)(\operatorname{div} \mathcal{Y})^{2} \\
& -\frac{2}{2 \varepsilon-R} \operatorname{tr} K \operatorname{div} \mathcal{Y}\left\langle{ }^{S} \nabla \mathcal{Y}, K\right\rangle .
\end{aligned}
$$

Therefore

$$
\begin{aligned}
& I=\left.\right|^{S} \nabla \mathcal{Y}-\left.\lambda K\right|_{g} ^{2}+\frac{(\operatorname{tr} K)^{2}}{|K|^{2}\left((\operatorname{tr} K)^{2}-|K|_{g}^{2}\right)}\left\langle{ }^{S} \nabla \mathcal{Y}, K\right\rangle^{2}+\frac{|K|_{g}^{2}}{(\operatorname{tr} K)^{2}-|K|_{g}^{2}}(\operatorname{div} \mathcal{Y})^{2} \\
& -\frac{2}{(\operatorname{tr} K)^{2}-|K|_{g}^{2}} \operatorname{tr} K \operatorname{div} \mathcal{Y}\left\langle{ }^{S} \nabla \mathcal{Y}, K\right\rangle .
\end{aligned}
$$

Hence we have

$$
\begin{aligned}
\left((\operatorname{tr} K)^{2}-|K|_{g}^{2}\right) I & =\left.\left((\operatorname{tr} K)^{2}-|K|_{g}^{2}\right)\right|^{S} \nabla \mathcal{Y}-\left.\lambda K\right|_{g} ^{2}+\frac{(\operatorname{tr} K)^{2}}{|K|_{g}^{2}}\left\langle{ }^{S} \nabla \mathcal{Y}, K\right\rangle^{2}+|K|_{g}^{2}(\operatorname{div} \mathcal{Y})^{2} \\
& -2 \operatorname{tr} K \operatorname{div} \mathcal{Y}\left\langle^{S} \nabla \mathcal{Y}, K\right\rangle \\
& =\left.\left((\operatorname{tr} K)^{2}-|K|_{g}^{2}\right)\right|^{S} \nabla \mathcal{Y}-\left.\lambda K\right|_{g} ^{2}+\frac{(\operatorname{tr} K)^{2}}{|K|_{g}^{2}}\left\langle{ }^{S} \nabla \mathcal{Y}, K\right\rangle^{2}+|K|_{g}^{2}(\operatorname{div} \mathcal{Y})^{2} \\
& \left.-2 \frac{\operatorname{tr} K}{|K|_{g}}|K|_{g} \operatorname{div} \mathcal{Y}{ }^{S} \nabla \mathcal{Y}, K\right\rangle .
\end{aligned}
$$

Therefore

$$
\left((\operatorname{tr} K)^{2}-|K|^{2}\right) I=\left((\operatorname{tr} K)^{2}-|K|^{2}\right)\left|S \nabla \mathcal{Y}-\frac{1}{|K|_{g}^{2}}\langle\nabla \mathcal{Y}, K\rangle K\right|_{g}^{2}+\left(|K|_{g} \operatorname{div} \mathcal{Y}-\frac{\operatorname{tr} K}{|K|_{g}}\left\langle{ }^{S} \nabla \mathcal{Y}, K\right\rangle\right)^{2} .
$$

Using this in (20), we get the following:

$$
\left.\int_{\Omega^{\prime}} \frac{1}{N}\right|^{S} \nabla \mathcal{Y}-\left.\frac{1}{|K|_{g}^{2}}\langle\nabla \mathcal{Y}, K\rangle K\right|_{g} ^{2} \mathrm{~d} M_{g}+\int_{\Omega^{\prime}} \frac{1}{(2 \epsilon-R) N}\left(|K|_{g} \operatorname{div} \mathcal{Y}-\frac{\operatorname{tr} K}{|K|_{g}}\left\langle{ }^{S} \nabla \mathcal{Y}, K\right\rangle\right)^{2} \mathrm{~d} M_{g}=0 .
$$

Since, by hypothesis, $2 \epsilon-\bar{R}>0$, then both integrands are non-negative; thus, for the equality to hold, both must equal zero. From the first integral, we get that

$$
{ }^{s} \nabla \mathcal{Y}=\frac{1}{|K|_{g}^{2}}\langle\nabla \mathcal{Y}, K\rangle K
$$

Taking traces we get

$$
\operatorname{div} \mathcal{Y}=\frac{1}{|K|_{g}^{2}}\langle\nabla \mathcal{Y}, K\rangle \operatorname{tr} K
$$

and multiplying by $|K|_{g}$ we obtain

$$
|K|_{g} \operatorname{div} \mathcal{Y}=\frac{\operatorname{tr} K}{|K|_{g}}\langle\nabla \mathcal{Y}, K\rangle
$$

which proves that (22) holds if and only if (23) holds. This shows that if $\mathcal{Y} \in \operatorname{ker} L$, then $\mathcal{Y}$ satisfies (23). The converse for this statement is also true. If we consider a field $\mathcal{Y}$ which satisfies (23), after some computations, we get that $L \mathcal{Y}=0$. So if (23) has only the trivial solution $\mathcal{Y}=0$, then $L$ is injective. 
At this point it is interesting to note that the curvature condition $2 \epsilon-R_{g}>0$ by itself does not pose any topological obstruction. This is because, for instance, if $\epsilon$ is continuous, then the compactness of $M$ implies that $\epsilon$ is bounded; hence, any metric on $M$ with scalar curvature which is more negative than $2 \min _{p \in M} \epsilon(p)$ satisfies this condition. That this last (stronger) condition can always be satisfied can be seen as a consequence of an important result in geometric analysis, which shows that on any compact $n$-dimensional manifold, $n \geq 3$, we can always choose a smooth metric $g^{\prime}$ such that $R\left(g^{\prime}\right)=-1$ (see Refs. 11 and 12). We then can always find a suitably rescaled metric $g$ satisfying $2 \epsilon-R_{g}>0$. Later on, using more subtle arguments, we will actually show that one can always find such metric within an initial data set satisfying the constraint equations. Note that, under our assumption, the Sobolev embedding theorems imply that we are assuming $\epsilon$ at least $C^{1}$, and thus, under our regularity hypotheses, this inequality does not impose any a priori restriction.

Using these results and applying the implicit function theorem, we have the following theorem.

Theorem 1. Suppose $\left(\psi_{0}, \beta_{0}\right) \in \mathcal{E}_{1} \times \mathcal{E}_{2}$ satisfies $\Phi\left(\psi_{0}, \beta_{0}\right)=0$. Then if $\pi$ is a definite operator at each point of $M, 2 \epsilon-R_{g}>0$ everywhere on $M$, and if for a given function $\mu$ on $M$ the equation

$$
{ }^{S} \nabla \mathcal{Y}=\mu K
$$

has only the solution $\mathcal{Y}=0, \mu=0$, then there are open neighbourhoods $\mathcal{V} \subset \mathcal{E}_{1}$ and $\mathcal{W} \subset \mathcal{E}_{2}$ of $\psi_{0}$ and $\beta_{0}$, respectively, and a unique mapping

$$
g: \mathcal{V} \rightarrow \mathcal{W}
$$

such that $\Phi(\psi, g(\psi))=0$ for all $\psi \in \mathcal{V}$.

Notice that this theorem shows that given an initial data set $\psi_{0} \in \mathcal{E}_{1}$ for which a solution $\beta_{0}$ of the reduced constraint equations $\Phi(\psi, \beta)=0$ exists, if the conditions stated in the theorem are satisfied, then for every $\psi \in \mathcal{V} \subset \mathcal{E}_{1}$, there is a unique solution of the reduced constraint equations. Then taking lapse defined as in (6), we get a solution of the full constraint equations. This answers our original question.

At this point, we would like to show that the conditions stated in the previous theorem are not too restrictive. By this we mean that, generically, there are solutions of the constraint equations satisfying all these conditions. With this in mind, notice that, given a solution $(g, K)$ for $(4)$ and (5) satisfying all the hypotheses of the previous theorem, we can use this solution obtained in the usual way, to obtain a reference solution for the TSP. In order to do this, just consider any given $N \in H_{s}, N>0$, and $\beta \in \mathcal{E}_{2}$ and take

$$
\dot{g}_{i j} \doteq 2 N K_{i j}+\left(\nabla_{i} \beta_{j}+\nabla_{j} \beta_{i}\right) .
$$

Then the set $((g, \dot{g}, \epsilon, S), \beta)$ gives a reference solution of the constraint equations. Thus, what we need to show is that the constraint equations (4) and (5) on a compact manifold $M$ always admit a solution $(g, K)$, satisfying all the hypotheses of the theorem. A first step in this direction is the following proposition.

Proposition 3. Suppose $(M, g)$ is an $n$-dimensional compact Riemannian manifold. Suppose that $(g, K)$ satisfy the constraint equations (4) and (5), where $K$ is a $(0,2)$ tensor field, and suppose that $2 \epsilon-R_{g}>0$ on $M$. Then, if the Ricci tensor on $M$ is negative definite, the equation ${ }^{S} \nabla \mathcal{Y}=\mu K$ has only the trivial solution $\mathcal{Y}=0$ and $\mu=0$.

Proof. Suppose $\mathcal{Y}$ and $\mu$ satisfy ${ }^{S} \nabla \mathcal{Y}=\mu K$. From the definition of the curvature tensor, we get the following:

$$
\begin{aligned}
R_{i j} \mathcal{Y}^{j} & =\nabla_{j} \nabla_{i} \mathcal{Y}^{j}-\nabla_{i} \nabla_{j} \mathcal{Y}^{j} \\
& =2 \nabla_{j}^{S} \nabla_{i} \mathcal{Y}^{j}-\nabla_{j} \nabla^{j} \mathcal{Y}_{i}-\nabla_{i}\left(\mu K_{j}^{j}\right) \\
& =2 \nabla_{j}\left(\mu K_{i}^{j}\right)-\nabla_{j} \nabla^{j} \mathcal{Y}_{i}-\nabla_{i}\left(\mu K_{j}^{j}\right) .
\end{aligned}
$$

Then we have

$$
R_{i j} \mathcal{Y}^{j} \mathcal{Y}^{i}=2 \mathcal{Y}^{i} \nabla_{j}\left(\mu K_{i}^{j}\right)-\mathcal{Y}^{i} \nabla_{j} \nabla^{j} \mathcal{Y}_{i}-\mathcal{Y}^{i} \nabla_{i}\left(\mu K_{j}^{j}\right)
$$


We can write this last expression in the following way, which is globally defined as

$$
\operatorname{Ric}_{g}(\mathcal{Y}, \mathcal{Y})=2 \operatorname{div}(\mu K) \cdot \mathcal{Y}-\langle\mathcal{Y}, \Delta \mathcal{Y}\rangle-\left\langle\nabla \operatorname{tr}_{g} \mu K, \mathcal{Y}\right\rangle
$$

In this last expression, $\Delta$ stands for the connection Laplacian defined by $\operatorname{tr}_{g} \nabla^{2}$. From the previous expression we get that

$$
\int_{M}\left(\operatorname{Ric}_{g}(\mathcal{Y}, \mathcal{Y})+\langle\mathcal{Y}, \Delta \mathcal{Y}\rangle+\left\langle\nabla\left(\mu \operatorname{tr}_{g} K\right), \mathcal{Y}\right\rangle-2 \operatorname{div}(\mu K) \cdot \mathcal{Y}\right) \mathrm{d} M_{g}=0
$$

Applying divergence theorem, we get

$$
\int_{M}\left(\operatorname{Ric}_{g}(\mathcal{Y}, \mathcal{Y})-|\nabla \mathcal{Y}|_{g}^{2}-\mu^{2}\left(\left(\operatorname{tr}_{g} K\right)^{2}-|K|_{g}^{2}\right)+\mu\langle K, \nabla \mathcal{Y}\rangle\right) \mathrm{d} M_{g}=0 .
$$

Now we will analyze the second and fourth terms in the integrand. In order to do this, we rewrite these expressions in the following way:

$$
\begin{aligned}
|\nabla \mathcal{Y}|_{g}^{2}-\langle\mu K, \nabla \mathcal{Y}\rangle & =\langle\nabla \mathcal{Y}, \nabla \mathcal{Y}\rangle-\left\langle{ }^{S} \nabla \mathcal{Y}, \nabla \mathcal{Y}\right\rangle=\left\langle\nabla \mathcal{Y}-{ }^{S} \nabla \mathcal{Y}, \nabla \mathcal{Y}\right\rangle \\
& =\left\langle{ }^{A} \nabla \mathcal{Y}, \nabla \mathcal{Y}\right\rangle=\left\langle{ }^{A} \nabla \mathcal{Y},{ }^{A} \nabla \mathcal{Y}\right\rangle \\
& =\left|{ }^{A} \nabla \mathcal{Y}\right|_{g}^{2},
\end{aligned}
$$

where

$$
{ }^{A} \nabla_{i} \mathcal{Y}_{j}=\nabla_{i} \mathcal{Y}_{j}-{ }^{S} \nabla_{i} \mathcal{Y}_{j}=\frac{1}{2}\left(\nabla_{i} \mathcal{Y}_{j}-\nabla_{j} \mathcal{Y}_{i}\right)
$$

Then, (27) can be rewritten as

$$
\int_{M}\left(\operatorname{Ric}_{g}(\mathcal{Y}, \mathcal{Y})-\left|{ }^{A} \nabla \mathcal{Y}\right|_{g}^{2}-\mu^{2}\left(2 \epsilon-R_{g}\right)\right) \mathrm{d} M_{g}=0
$$

Thus, if $\mathrm{Ric}_{g}$ is negative definite, then the integrand is non-positive. Hence in order for the last equality to hold, each term in the integrand has to equal zero. So the only possible $\mathcal{Y}$ and $\mu$ which can satisfy ${ }^{S} \nabla \mathcal{Y}=\mu K$ under these geometric conditions are $\mathcal{Y}=0, \mu=0$.

This proposition implies that, given a solution of the constraint equations $\left(g_{0}, K_{0}\right)$ satisfying $2 \epsilon-R>0$, then if $\pi$ is a definite operator on $M$ and $\operatorname{Ric}_{g_{0}}$ is negative definite, then the linearization $L=\left.\frac{\delta \Phi}{\delta \beta}\right|_{\left(\psi_{0}, \beta_{0}\right)}$ is an isomorphism and Theorem 1 applies. A 3-dimensional version of the previous proposition was shown in Ref. 1.

We will now show that any compact $n$-dimensional manifold admits a solution of the constraint equations satisfying all the hypotheses of Theorem 1 . The first step in this direction is to look for a solution of the constraint equations of the form $(h, \alpha h)$, with $h$ being a Riemannian metric and $\alpha$ a positive constant. That is, we are considering $K=\alpha h$ from the beginning. We will restrict ourselves to solutions of (4) and (5) with $S=0$, i.e., with zero momentum density. With this set up, Eq. (5) is automatically satisfied and we just need to consider Eq. (4), which, under these conditions, becomes the following equation, which is posed for $h$ :

$$
R_{h}=2 \epsilon-\alpha^{2} n(n-1) .
$$

In order to guarantee the existence of solutions for (29), we will appeal to the following wellestablished theorem:

Theorem 2. Let $M$ be a $C^{\infty}$ compact manifold of dimension $n \geq 3$. If $f \in C^{\infty}(M)$ is negative somewhere, then there is a $C^{\infty}$ Riemannian metric on $M$ with $f$ as its scalar curvature.

This theorem was proved by Kazdan and Warner, ${ }^{9}$ and its proof can also be found in Ref. 10. Using this theorem, we see that if the right-hand side of (29) is negative somewhere, then, for smooth sources $\left(\epsilon \in C^{\infty}\right)$, we have that (29) always admits a smooth solution. In order to guarantee this last condition, just take $\alpha^{2}>\min \frac{2 \epsilon}{n(n-1)}$. A solution constructed in this way satisfies two of the three conditions required by Theorem 1, that is, it satisfies the following: 
- $2 \epsilon-R_{h}>0$, which comes from (29).

- $\pi$ is negative definite, since from $K=\alpha h$ we get that $\pi=\alpha(1-n) h$.

In this context, the last condition of Theorem 1 becomes the statement that $h$ does not admit conformal Killing fields. We will show that we can always find a solution $h$ of (29) with this property. In order to do this, we need to make a remark on how Theorem 2 is proved (see, for example, Ref. 10 Chap. 6). The proof of this theorem begins with the statement that we can choose on $M$ a Riemannian metric $g^{\prime}$, with $R_{g^{\prime}}=-1$, which is something known from Ref. 11, and then one finds a conformal metric to $g^{\prime}$ satisfying the theorem. In fact, it is shown that $h$ has the following form:

$$
h=\left(\phi^{-1}\right)^{*}\left(u^{\frac{4}{n-2}} g^{\prime}\right),
$$

where $u$ is a positive function and $\phi$ is a suitably chosen diffeomorphism. In this process, we claim that we can choose $g^{\prime}$ without conformal Killing fields. We support this claim using the results shown by Lohkamp in Ref. 12. There, it is shown that every manifold $M$ of dimension $n \geq 3$ admits a complete metric with negative definite Ricci tensor. As a corollary of this theorem, it is shown that, starting from such a Riemannian metric $g$ on $M$ with negative definite Ricci tensor, we can find a conformal metric $g^{\prime}=v^{\left(\frac{4}{(n-2)}\right)} g$, such that $R\left(g^{\prime}\right)=-1$. In this way, using this metric as the starting point in the proof of Theorem 2, we get that the metric $h$ solving (29) has the following form:

$$
h=\left(\phi^{-1}\right)^{*}\left(u^{\frac{4}{n-2}} v^{\frac{4}{n-2}} g\right),
$$

where $g$ has a negative definite Ricci tensor. We now have the following:

\section{Proposition 4. The solution (31) obtained for (29) does not admit conformal Killing fields.}

Proof. It is a well-known fact that, on compact manifolds, metrics with negative definite Ricci tensor do not admit conformal Killing fields (see, for instance, Ref. 3 Chap. 7), which is the case of the metric $g$. Now, imagine that $h$ admitted a conformal Killing field $Y \in \Gamma(T M)$, that is, $£_{Y} h=\lambda h$, for some $\lambda \in C^{\infty}(M)$, where $f_{Y} h$ is the Lie derivative of $h$ with respect to $Y$. From (31) we get that

$$
g=(u v)^{\frac{-4}{n-2}} \phi^{*}(h) \doteq \mu \phi^{*}(h) .
$$

Define $X \doteq \phi_{*}^{-1} Y \in \Gamma(T M)$ so that $Y=\phi_{*} X$. We claim that under these conditions $X$ is a conformal Killing field of $g$. To check this, we have to compute the Lie derivative of $g$ with respect to $X$, that is,

$$
f_{X} g=X(\mu) \phi^{*}(h)+\mu f_{X}\left(\phi^{*} h\right) .
$$

Using that $£_{X}\left(\phi^{*} h\right)=\phi^{*}\left(£_{Y} h\right)$ (see Ref. 13) and the fact that $Y$ is a conformal Killing field for $h$, then we get that

$$
f_{X} g=(X(\log \mu)+\lambda \circ \phi) g
$$

which shows that $X$ is a conformal Killing field for $g$. But this contradicts the fact that $g$ has a negative definite Ricci tensor, thus $h$ cannot admit conformal Killing fields.

Thus, we conclude that the solution we have constructed for the constraint equations satisfies all the hypotheses of Theorem 1 and therefore can be used as a reference solution. Then we can state the following theorem.

Theorem 3. Any smooth compact $n$-dimensional manifold $M, n \geq 3$, admits a smooth solution $(N, \beta)$ of the constraint equations (4) and (5) with $S=0$, with prescribed smooth free data $\psi$ $=\left(g, \dot{g}, \epsilon_{\alpha}, 0\right) \in \mathcal{E}_{1}$, such that in an $\mathcal{E}_{1}$-neighbourhood of $\psi$ the TSP is well-posed .

\section{FINAL REMARKS}

In this paper, we have been able to show the validity of the main results presented in Ref. 1 in arbitrary dimensions $(n \geq 3)$. Specifically we have shown that Wheeler's thin sandwich conjecture is true under certain geometrical conditions in all these cases. As a novelty, we have also been able to establish that the geometric hypotheses needed to prove this result can always be satisfied in the case 
of zero momentum density, and thus that, in these cases, there is an open subset in the space of possible initial data for the constraint equations where the thin sandwich problem is well-posed. These are interesting results describing the space of solutions of the constraint equations in arbitrary dimensions. These types of results are relevant in the study of the Cauchy problem for higher dimensional theories of gravity, and they also give us a better understanding of the Superspace picture for space-time in this context. In contrast to the usual approach to the constraint equations, where the structure of the space of solutions and its relation to properties of the evolving space-time are something we understand quite well (see, for instance, Refs. 3, 14, and 15), most of these problems require further study in the context of the Superspace picture for space-time.

Finally, it is worth pointing out that even though we have not followed the strategy presented in Ref. 8 regarding the study of the TSP, the results obtained therein suggest further research in the investigation of the TSP in, perhaps, more physically realistic situations, since in this work matter fields are included into the picture. Moreover, the results obtained in Ref. 8 offer some techniques which could complement the ones presented here, such as the global uniqueness theorem which is presented there (following the lines of Ref. 7), and a method to construct families of reference solutions once one solutions satisfying the geometric restrictions needed is obtained. Thus, we regard the combination of the two strategies as a starting point for future research in this area.

\section{ACKNOWLEDGMENTS}

R.A. and C.R. would like to thank CNPq and CLAF for financial support. J.L. would like to thank CNPq and FUNCAP/CNPq/PRONEX for financial support. We thank the referee for valuable comments and suggestions.

${ }^{1}$ R. Bartnik and G. Fodor, "On the restricted validity of the thin sandwich conjecture," Phys. Rev. D 48, 3596-3599 (1993).

${ }^{2}$ Y. Choquet-Bruhat and R. Geroch, "Global aspects of the Cauchy problem in general relativity," Commun. Math. Phys. 14, 329 (1969).

${ }^{3}$ Y. Choquet-Bruhat, General Relativity and the Einstein Equations (Oxford University Press, Inc., New York, 2009).

${ }^{4}$ H. Ringström, "The Cauchy problem in general relativity" (European Mathematical Society, Germany, 2009).

${ }^{5}$ D. Giulini, "The superspace of geometrodynamics," Gen. Relativ. Gravitation 41, 785 (2009).

${ }^{6}$ C. W. Misner, K. S. Thorne, and J. A. Wheeler, Gravitation (W. H. Freeman and Company, San Francisco, 1973).

${ }^{7}$ E. P. Belasco and H. C. Ohanian, "Initial conditions in general relativity: Lapse and shift formulation," J. Math. Phys. 10, 1503 (1969).

${ }^{8}$ D. Giulini, "The generalized thin-sandwich problem and its local solvability," J. Math. Phys. 40, 2470 (1999).

${ }^{9}$ J. Kazdan and F. Warner, "Scalar curvature and conformal deformation of Riemannian structure," J. Differ. Geom. 10, 113-134 (1975).

${ }^{10}$ T. Aubin, Some Non-Linear Problems in Riemannian Geometry (Springer, Berlin, 1998).

${ }^{11}$ T. Aubin, "Metriques riemanniennes et courbure," J. Differ. Geom. 4, 383-424 (1970).

12 J. Lohkamp, "Metrics of negative Ricci curvature," Ann. Math. 140, 655-683 (1994).

${ }^{13}$ J. E. Marsden, T. Ratiu, and R. Abraham, in Manifolds, Tensor Analysis, and Applications (Springer-Verlag Publishing Company, New York, 2007), Chap. 6.

${ }^{14}$ A. E. Fischer, J. E. Marsden, and V. Moncrief, “The structure of the space of solutions of Einstein's equations. I. One killing field," Annales de l'I.H.P. Physique Théorique 33(2), 147-194 (1980).

${ }^{15}$ R. Beig, P. T. Chruściel, and R. Schoen, “KIDs are not generic," Ann. Henri Poincaré 6, 155 (2005). 